\title{
Perceptual Dynamic Range for In-Camera Image Processing
}

Praveen Cyriac

praveen.cyriac@upf.edu

David Kane

david.kane@upf.edu

Marcelo Bertalmío

marcelo.bertalmio@upf.edu
Department of Information and Communication Technologies, Universitat Pompeu Fabra,

Barcelona, Spain
Image sensors in digital cameras capture values which are proportional to light intensity and within a range of between 3-4 orders of magnitude. These values are typically passed through a non-linearity prior to quantisation to a range of around 2 orders of magnitude. The purpose of the non-linearity is to ensure that the quantisation rate is approximately proportional to human sensitivity (see [2] and references therein). The problem of reducing the dynamic range of an image while preserving the perceived detail, is known as tone mapping. Digital cameras generally do this by means of gamma correction, which is a very simple, image independent approach, designed to produce good results on average.

We propose an adaptive in-camera non-linearity that ensures that the detail and contrast visible in the processed image match closely with the perception of the original scene. The method has been developed to emulate basic properties of the human visual system including contrast normalisation and the efficient coding of natural images via adaptive processes which ensures that the output values are more evenly distributed over the available range. The transformations applied to the image are based on natural image statistics, psychophysical data and neurophysiological models. We have followed the same approach as Ferradans et al. in [4] and our method consists of two main stages, a global one followed by a local contrast enhancement one as shown in Figure 1:

1. Using natural image statistics, a function $\gamma$ is defined by estimating its parameters $\left(\gamma_{L}, \gamma_{H}\right.$ and $\left.M\right)$ from the cumulative histogram of the intensity image in log-log coordinates.

$$
\gamma(I)=\gamma_{H}+\left(\gamma_{L}-\gamma_{H}\right)\left(1-\frac{I^{n}}{I^{n}+M_{l i n}^{n}}\right),
$$

This function $\gamma$ is used to perform a transform of the intensity values (Eq. (2)) in a manner that complies with users' preference data as obtained through psychophysical tests [6].

$$
I_{1}(x)=(I(x))^{\gamma(I(x))} .
$$

2. The output of the previous stage is passed through an additional contrast normalisation procedure that replicates efficient coding behaviour that occurs both in the retina and cortical areas of the human visual system.

$$
O(x)=\mu(x)+\left(I_{1}(x)-\mu(x)\right) * k / \sigma,
$$

where $x$ is a pixel, $I_{1}(x)$ is the value at pixel $x$ computed at the previous stage of our method, $\mu(x)$ is the local mean of $I_{1}, k$ is a constant, $\sigma$ is the standard deviation of $I_{1}$, and $O(x)$ is the final output value of our method for pixel $x$.

Both equations (Eqs. (2) and (3)) are applied separately to each of the red, green and blue colour channels, which have previously been normalised into the range $[0,1]$ and where values above the 99 percentile have been clipped.

For video sequences, applying our method to each frame separately may result in flickering artefacts due to the possibility of a sudden change in the tone curve from one frame to the next. Therefore for video we adopt a two pass approach. In the first pass, we estimate $\gamma_{L}, \gamma_{H}$ and $M$ for each frame separately, then we apply a temporal low pass filter to these values. In the second pass, we apply our method (Eqs. (2) and (3)) with the new parameters.

In this paper we illustrate the potential of our method by comparing the regular JPEG output of the camera versus the result of applying our method to the corresponding RAW image. The results are illustrated

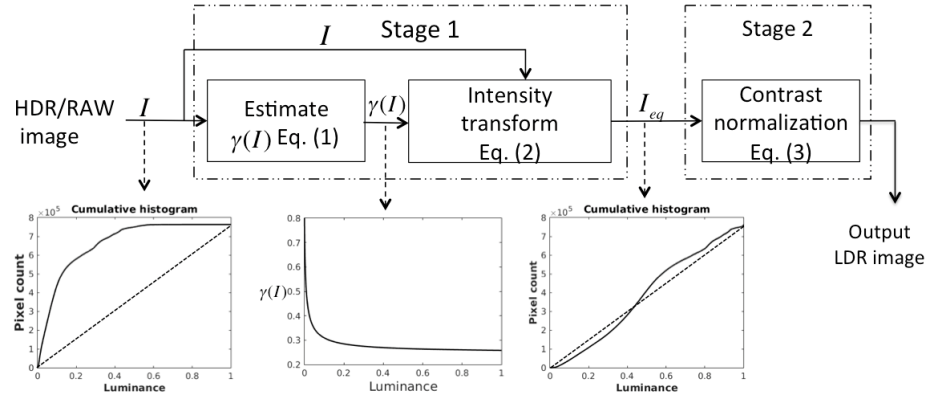

Figure 1: Block diagram of the proposed method.

with images from consumer, smartphone and cinema cameras. The results have natural appearance, enhanced contrast and absence of halos, spurious colours or visual artefacts of any kind. Our method can also be used offline as a tone mapping operator, applied to HDR images and we illustrated our result on two HDR data set [3][5]

We also perform a quantitative evaluation of our method. We use the Fairchild dataset [3] of HDR images and compare our algorithm with six state-of-the-art tone mapping operators, using the metrics DRIM [1] and TMQI [8]. According to DRIM, the amount of distortions produced by our approach matches that of Mantiuk et al. [7] and is significantly less than the error introduced by all other tested methods. In terms of TMQI, our method ranks as the best in terms of overall quality $(\mathrm{Q})$, and matches to Mantiuk et al. [7] for structural fidelity (S). It is worth noting that our results have a very high naturalness $(\mathrm{N})$ index.

The proposed approach is of low computational complexity and therefore it is a very good candidate for real-time applications.

[1] Tunç Ozan Aydin, RafałMantiuk, Karol Myszkowski, and Hans-Peter Seidel. Dynamic range independent image quality assessment. SIGGRAPH '08, pages 69:1-69:10, 2008.

[2] Marcelo Bertalmío. Image Processing for Cinema. CRC Press, 2014.

[3] Mark D Fairchild. The hdr photographic survey. In Color and Imaging Conference, volume 2007, pages 233-238, 2007.

[4] Sira Ferradans, Marcelo Bertalmio, Edoardo Provenzi, and Vicent Caselles. An analysis of visual adaptation and contrast perception for tone mapping. Pattern Analysis and Machine Intelligence, IEEE Transactions on, 33(10):2002-2012, 2011.

[5] Jan Froehlich, Stefan Grandinetti, Bernd Eberhardt, Simon Walter, Andreas Schilling, and Harald Brendel. Creating cinematic wide gamut hdr-video for the evaluation of tone mapping operators and hdr-displays, 2014.

[6] David Kane and Marcelo Bertalmío. Is there a preference for linearity when viewing natural images? In SPIE/IS\&T 2015 Image Quality and System Performance XX, 2015.

[7] RafałMantiuk, Scott Daly, and Louis Kerofsky. Display adaptive tone mapping. ACM Trans. Graph., 27(3):68:1-68:10, August 2008.

[8] H. Yeganeh and Zhou Wang. Objective quality assessment of tonemapped images. Image Processing, IEEE Transactions on, 22(2): 657-667, Feb 2013. 